\title{
Spatial location of correlations in a random distributed feedback Raman fiber laser
}

\author{
I. D. Vatnik, ${ }^{1,2 *}$ O. A. Gorbunov, ${ }^{1,2}$ S. Sugavanam, ${ }^{3}$ D. V. Churkin ${ }^{1,2}$, E. V. \\ PODIVILOV ${ }^{1,2}$ \\ ${ }^{1}$ Novosibirsk State University, Novosibirsk 630090, Russia \\ ${ }^{2}$ Institute of Automation and Electrometry SB RAS, Novosibirsk, 630090, Russia \\ ${ }^{3}$ Aston Institute of Photonic Technologies, Aston University, Birmingham, B4 7ET, UK \\ *Corresponding author ilya.vatnik@gmail.com
}

Received XX Month XXXX; revised XX Month, XXXX; accepted XX Month XXXX; posted XX Month XXXX (Doc. ID XXXXX); published XX Month XXXX

\begin{abstract}
Nonlinear interactions between different components of multiwavelength radiation is one of the main processes shaping properties of quasi-CW fiber lasers. In random fiber lasers nonlinear influence may be more complicated as there are no distinct longitudinal modes in radiation because of random nature of the feedback. In the present paper, we experimentally characterize internal correlations in the radiation of multiwavelength random distributed feedback fiber laser. Analysis of Pearson correlation functions allows us to spatially locate the area over the fiber laser length in which correlations more likely to occur. This in turn leads us to the conclusion about the main mechanism of spectral correlations - relative intensity noise transfer from the pump wave.
\end{abstract}

http://dx.doi.org/10.1364/OL.99.099999

Spectral correlations in the radiation of fiber lasers have attracted a lot of attention for the last decade as they bring a new insight on formation of multimode generation, in particular multiwavelength generation. Knowledge of interplay between different spectral components in the output radiation is crucial for both fundamental and applied issues such as analysis of laminar-turbulent transition in radiation of a fiber laser [1,2], a more general problem of intermittency in integrable turbulence [3], a problem of extreme events and correlations appearing during propagation of light along fiber [4-7], reviewing a unified theoretical formulation of statistical nonlinear optics on the basis of the wave turbulence theory [8] etc. In particular, study of spectral correlations may shed light on the physics governing generation in random distributed feedback (RDFB) fiber lasers.
Experimentally, there are few approaches to study correlations in a long fiber lasers quasi-CW generation to which class the RDFB laser belongs. The powerful tool is to measure a time dynamics of the output radiation intensity and then to analyze the intensity probability density functions (PDF) and autocorrelation functions [9-11]. The correlations can be revealed as heavy-tailed or low-tailed deviations for intensity PDF, being measured for the overall radiation by asynchronous optical sampling technique [12] or for the spectrally-preselected part of the radiation [13].

Another possibility to get an access to correlations in the radiation is to measure an instantaneous spectrum, and then analyze spectral correlations within an ensemble of different spectra, which has been done for the RDFB fiber laser in Ref. [14]. The long-living correlations were found on a time scale of hundred milliseconds and are attributed to stimulated Brillouin scattering (SBS), which is known to play a role only right above the threshold [15]. In those measurements the acquisition speed was limited down to $1 \mathrm{kHz}$, which does not correspond to the intrinsic fast dynamics in the laser. The similar approach was implied to signature turbulence regime in a continuouswave-pumped erbium-based random fiber laser with random Bragg gratings [16], which is characterized by slow spectral dynamics.

Apart from SBS, in long Raman fiber lasers there are a number of processes that may be responsible for spectrum formation. First, Kerr nonlinearity-induced four-wave mixing (FWM) could potentially couple amplitudes of different waves that results in correlations. It was predicted analytically that weak Kerr nonlinearity may cause deviation of intensity PDF from exponential form [17]. Another possible mechanism is cross-phase modulation (XPM), nonlinear interaction of a pump wave with generation waves through the same Kerr effect. 
Finally, fast response of the Raman scattering process could result in substantial contribution of the relative intensity noise (RIN) transfer from multimode quasi-CW pump wave to generation wave. In particular, pump-toStokes noise transfer defines the generation width of the Raman fiber lasers generation spectrum near the generation threshold [18]. All these processes may lead to appearance of correlations that reveal itself indirectly in total intensity PDF, but are too fast and thus cannot be captured in instantaneous spectral measurements.

In this Letter we implement another approach that brings more information on correlations and underlying processes. We propose to study correlations by direct simultaneous measurements of intensity dynamics of different spectral components and further analyze the Pearson correlation function. The dependence of temporal delay of correlation peak over the wavelength difference between correlated lines allows us to spatially locate the correlation area over the fiber laser length. This, in turn, has allowed us to prove that correlations in the random fiber laser under study are brought by relative intensity noise transfer from the pump wave.

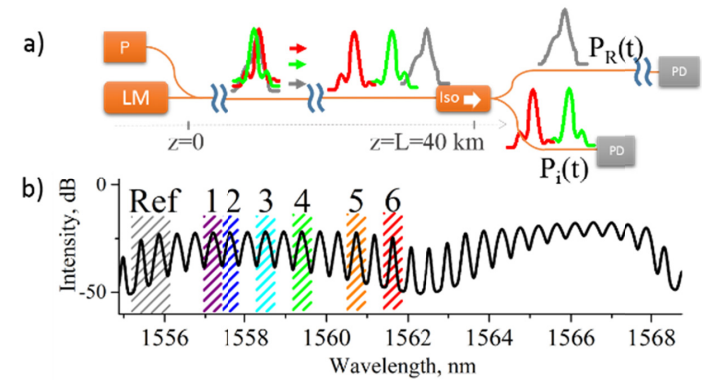

Fig. 1. (a) Experimental setup. LM - fiber loop mirror with a Lyot filter [19]. P -a pump laser at $1455 \mathrm{~nm}$. Iso - an isolator placed at the laser output followed by a coupler, spectral filters (not shown) and photodiodes PD. $P_{R}$ is a reference line channel and $P_{i}$ is a channel for all other lines.(b) The generation spectrum for pump power $P=2.3 \mathrm{~W}$ well above the threshold and positions of spectral filters.

Experimental setup is schematically shown on Fig. 1a. A laser under study is a multiwavelength RDFB Raman fiber laser [19,20]. The laser comprises $L=40 \mathrm{~km}$ of SMF-28 fiber pumped in forward direction at $1455 \mathrm{~nm}$. A generation spectrum well above the threshold consists of separate lines with a spectral width of $\sim 0.5 \mathrm{~nm}$. Output radiation is launched into two optical channels via $3 \mathrm{~dB}$ coupler. Light in each channel is then passed through a tunable spectral filter to obtain a single spectral line, intensity dynamics of which is registered by a fast $(50$ $\mathrm{GHz}$ ) photodetector and a digital oscilloscope (16 GHz electrical bandwidth, $40 \mathrm{GS} / \mathrm{s}$ sampling rate). We set width and position of the first spectral filter fixed at the left spectral end (see Fig. 1b). To obtain enough power for consistent registration it was necessary to cover two edge lines at wavelengths $1555.4 \mathrm{~nm}$ and $1555.8 \mathrm{~nm}$ with the spectral filter - hereafter they are referred to as a reference line. Position and width of the filter in the second channel referred to as signal was adjusted to successively cover other spectral lines at wavelengths $1557.1 \mathrm{~nm}, 1557.6 \mathrm{~nm}, 1558.5 \mathrm{~nm}, 1559.4 \mathrm{~nm}, 1560.7 \mathrm{~nm}$, and $1561.6 \mathrm{~nm}$ - at Fig. 1b they are numbered from 1 to 6 .

Typical temporal intensity dynamics in both channels are stochastic (see Fig. 2a). The intensity probability density function for the multiwavelength random laser deviates from pure exponential shape thus internal correlations should exist [19]. To uncover possible interline correlations we use Pearson correlation coefficient (PCC), widely used to describe a linear correlation between two random values [21]:

$$
\operatorname{PCC}(\tau)=\frac{\left\langle\delta I_{R}(t) \delta I_{i}(t+\tau)\right\rangle_{t}}{\left\langle\delta I_{R}^{2}\right\rangle_{t}\left\langle\delta I_{i}{ }^{2}\right\rangle_{t}}
$$

where $\delta I_{R}=\left(I_{R}-\left\langle I_{R}\right\rangle_{t}\right), \delta I_{i}=\left(I_{i}-\left\langle I_{i}\right\rangle_{t}\right)$ are intensity variations of reference line and a signal line around their average values correspondingly, $\langle\ldots\rangle_{t}$ is time averaging.
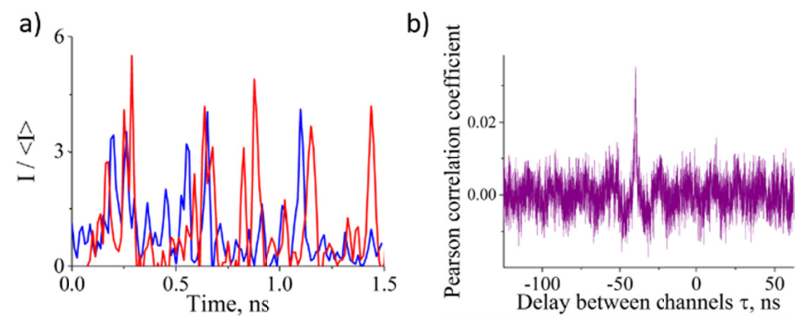

Fig. 2. (a) Temporal evolution of simultaneously measured intensity dynamics for reference line and line \#1. (b) Pearson correlation coefficient (PCC) as a function of temporal delay $\tau$ for the line \#1 and the reference line.

$\operatorname{PCC}(\tau)$ has been calculated in a wide range of possible delays (up to $2 \mu$ s delays) as it is a priori unknown what kind of correlations could be found in a signal. Obviously, for arbitrary time shift signals in two channels are initially non-simultaneous and consequently should be statistically independent (PCC $\approx 0$ ). If there are any correlations between signals, the Pearson correlation coefficient gets non-zero value. First, we calculated $\operatorname{PCC}(\tau)$ for the reference line and the line at $1557.1 \mathrm{~nm}$ (line \#1 as numbered at fig $1 \mathrm{~b}$ ). It has a single distinct maximum at $\tau_{0}$ $=-44 \mathrm{~ns}$ (Fig. $2 \mathrm{~b}$ ). The negative value means that it takes longer for the light in the reference line to reach the oscilloscope. Note that the exact value of the $\tau_{0}$ does not have any specific meaning. Indeed, there is an uncertain delay between two signals approaching the photodiodes in two channels because of unequal lengths of fiber coming from the output coupler to each photodiode. There are no other pronounced maxima or minima in $\operatorname{PCC}(\tau)$ function in the whole $2 \mu$ s range of temporal delay $\tau$. The value of PCC at the maximum is less than 0.04 that indicates quite weak correlations between these two spectral components. However, the intensity of the correlation peak is much larger than the measured level of uncorrelated background which is around 0.005, Fig.3a. We would like to note that spectral filtering within a limited bandwidth could potentially results in artefact correlations. We built a numerical model simulating an impact on spectral filtering 
on completely stochastic intensity dynamics, and found that spectral filtering accounts for the correlations value below 0.001 for experimental values of spectral filter bandwidth of $0.5 \mathrm{~nm}(65 \mathrm{GHz})$.

We repeated calculations of PCC for all the lines 1-6 paired with the reference line. We have found $\operatorname{PCC}(\tau)$ for all the pairs to have a peak, thus spectral components separated by more than $6 \mathrm{~nm}$ turned out to be correlated, Fig. 3a. A value of PCC at the maximum decreases with increase of spectral distance between the reference line and a line under study. Moreover, $\operatorname{PCC}(\tau)$ maximum is located at different times for different lines, so temporal shift $\tau_{0}$ depends on line number (i.e. wavelength), Fig. $3 \mathrm{~b}$.
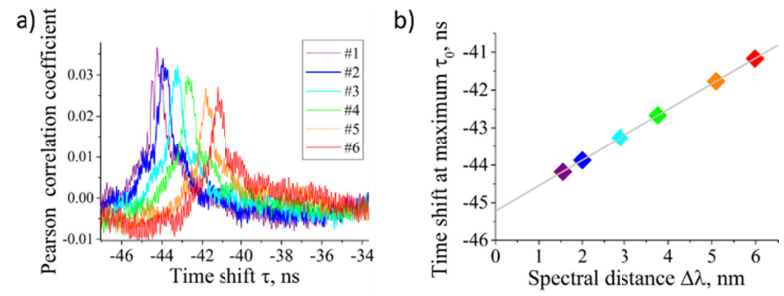

Fig. 3.(a) Pearson correlation coefficient (PCC) between the $i^{\text {th }}$ line and the reference line as function of temporal delay $\tau$ (b) PCC peak position $\tau_{0}$ as function of spectral distance $\Delta \lambda$ between $i^{\text {th }}$ line and the reference line. Grey line is linear fit with slope $0.678 \mathrm{~ns} / \mathrm{nm}$. Colour code on panels (a) and (b) is the same and correspond to the line number (see legend to panel (a) for correspondance).

To describe variety of delays between channels for different line pairs we propose a following model. We assume that two waves at each pair interact locally (which subsequently leads to correlations in their intensities) at the same confined area within the fiber, and then further propagate without interactions. Then the main contribution to the time delay $\tau_{0}$ for different pairs should arise from difference of propagation speed for waves at different wavelengths.

Within these assumptions, dependence of time delay $\tau_{0}$ on wavelength becomes evident. Indeed, as SMF-28 fiber has an anomalous dispersion around $1.55 \mu \mathrm{m}$, radiation at larger wavelengths (i.e. more distant from the reference wavelength) propagate with a smaller group velocity than the radiation at the reference spectral line. Consequently, for lines with larger wavelengths and larger numbers (as numbered at the Fig. $1 \mathrm{~b}$ ), the increase of the delay $\tau_{0}$ is observed (Fig. 3b). Moreover, as the group velocity value depends linearly on the wavelength in the considered small range of wavelengths, the resulted dependence of $\tau_{0}$ on wavelength should be linear, as it is observed experimentally, Fig. 3b, grey line.

From experimental measurements of $\tau_{0}$ we can define the propagation length of waves from the point of their interaction by:

$$
\tau_{0}\left(\lambda_{i}\right)=-t_{0}+L_{e f f} D(\Delta \lambda) ; \quad L_{e f f}=\frac{1}{D} \frac{d \tau_{0}}{d \Delta \lambda}
$$

where $L_{e f f}$ - distance the waves propagate since the interaction till they leave the laser cavity, $t_{0}$ - temporal delay between channels, caused by all factors except dispersion of group velocities within the fiber, $D$ is the dispersion parameter of the SMF-28 fiber, $\Delta \lambda$ is absolute value of difference between reference wave wavelength $\lambda_{R}$ and signal wavelength $\lambda_{i}$. We used the standard value for dispersion parameter $D$ indicated in technical specification for SMF-28 fiber, $D=16.3 \pm 0.5 \mathrm{ps} / \mathrm{nm} / \mathrm{km}$. From experimental measurements of $\tau_{0}$ we define the coefficient $d \tau_{0} / d \Delta \lambda=0.678 \pm 0.016 \mathrm{~ns} / \mathrm{nm}$, which gives length $L_{\text {eff }}=41 \pm 2 \mathrm{~km}$. In this way we define the spatial location of the correlations in the random DFB fiber laser as the laser length in our particular case is $L=40 \mathrm{~km}$, interactions occur near the point $z=0$, where the pump wave enters the fiber.
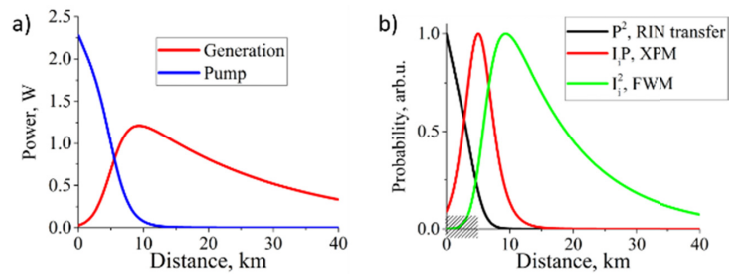

Fig. 4. (a) Longitudinal distributions of pump power $P(z)$ and total generation power $I(z)$ along the fiber length $z$. (b) Spatial distribution of correlation strength $\rho(z)$ corresponding to pump-to-Stokes intensity noise transfer $\left(\sim P^{2}\right)$, cross-phase modulation between generation wave and pumpe wave $\left(\sim I_{i} P\right)$ and four-wave mixing within the generation wave $\left(\sim I_{i}^{2}\right)$ calculated from the power balance model. Grey shaded area is an estimated area where spectral correlations arise.

Further we consider three different processes that could lead to correlations: pump-to-Stokes relative intensity noise (RIN) transfer, cross-phase modulation (i.e. interaction of pump wave with generation wave) and fourwave mixing (i.e. interaction of different Stokes waves between them). The strength of each process is proportional to the intensities of interacting waves $P^{2}, P \cdot I_{i}, I_{i}^{2}$, correspondingly to RIN transfer, XPM, FWM. It is known that both the pump power, $P(z)$, and generation intensity, $I(z)$, varies strongly over the fiber length, $z$ [22]. This potentially allows us to determine which process contributes mostly to the correlations that are found to be spatially located near $z=0$. Indeed, one can calculate the spatial distribution of the interaction rate over the fiber length for each process $\rho(z) \sim P^{2}(z)$, $P(z) I_{i}(z), I_{i}^{2}(z)$. To do this we use a power balance model [22], and calculate the longitudinal distribution of both the pump power and total generation power, Fig. 4a. We verify the model and parameters used by calculating the output generation power and comparing its value with experimental data. Then we take into account that the intensity of each spectral line $I_{i}$ is approximately equal to total power, divided by total number of lines in the spectrum: $I_{i} \sim I / N_{L}, I_{R} \sim 2 I / N_{L}$, with $N_{L}=28$, as the generation spectrum has a high level flatness $[19,20]$. The calculated distributions of the correlation strength $\rho(z)$ are highly non-uniform over the fiber length, Fig. 4b. The only process which can result in high interactions rate near $z=0$ is the pump-to-Stokes RIN transfer. XPM and 
FWM would result in different location of the interaction area and, subsequently, in different dependence of the position of the correlation peak on time delay.

Designating the dominant kind of interactions we can theoretically estimate the width $\Delta \tau_{0}$ of the correlation function $\operatorname{PCC}(\tau)$. The first reason of the non-zero width is that interactions can occur at different points over the fiber length: waves which interact closer to the point $z=0$ provides bigger delay $\tau_{0}$ in comparison to waves interacting at larger $z$, see Eq. (2). The corresponding contribution to the width $\Delta \tau_{0}$ should vary for different pairs can be estimated as $\delta \beta_{R i} L_{P}$, where $\delta \beta_{R i}=D \Delta \lambda$ is the difference of group velocities between reference and signal $i^{\text {th }}$ wave, and $L_{P}$ is the length of the fiber where the average pump power is high and correlations are gained. We estimate $L_{P} \sim 5 \mathrm{~km}$ from longitudinal pump power distribution (see Fig. 4b), which corresponds to the correlation function width of $\delta \beta_{R i} L_{P} \sim 0.1-0.5$ ns for wavelengths $\Delta \lambda=1.8-6.2 \mathrm{~nm}$.

The next contribution to the correlation function width $\Delta \tau_{0}$ arises as the correlations between different Stokes waves are induced by the same pump wave fluctuation $\delta P$ but in different places within the amplification region $\left[0, L_{P}\right]$. Indeed, a typical pump wave fluctuation propagates a distance $L_{D}=\delta t^{2} / \beta_{2}(P) \sim 0.1 \mathrm{~km}$ before it considerably broadens due to dispersion. Here $\delta t$ is a temporal width of pump wave fluctuation estimated as 1 ps from the pump wave spectral width of $5 \mathrm{~nm}, \beta_{2}=13 \mathrm{ps}^{2} / \mathrm{km}$ is dispersion coefficient for pump wavelength. Thus a pump fluctuation may couple two Stokes waves that are not only at the same point but $0.1 \mathrm{~km}$ apart. This results in an additional delay $\Delta \tau_{0}=\delta \beta_{P S} L_{D} \sim 0.1$ ns determined by a difference in group velocities of pump and Stokes waves $\delta \beta_{P S}=1.3 \mathrm{~ns} / \mathrm{km}$.

Finally, we take into account that the reference channel in our experiment actually consists of two lines with difference of propagation constants $\delta \beta_{R}=8 \mathrm{ps} / \mathrm{km}$ that also contributes to $\Delta \tau_{0}$ by $\delta \beta_{R} L \sim 0.3$ ns.

Thus the estimated total width of correlation function is

$$
\Delta \tau_{0}=\delta \beta_{R_{i}} L_{P}+\delta \beta_{P S} L_{D}+\delta \beta_{R} L \sim 0.5 \div 0.9 \mathrm{~ns}
$$

that qualitatively agrees with experimentally observed widths less or equal to 1 ns, Fig 3a.

Note that small anticorrelations are also present in $\operatorname{PCC}(\tau)$ function (see Fig. 2b). These negative correlations may be attributed to four-wave mixing that enables power exchange between reference and signal channels. As the FWM may occur with same small but non-zero probability almost everywhere within the laser cavity (see Fig. 4b), the anticorrelations appear as a broad substrate in $\operatorname{PCC}(\tau)$. Indeed, the similar estimations as above gives several times larger width of PCC. Relatively small contribution of FWM into the net value of correlations could be explained by a large nonlinear length $L_{N L}=1 / \gamma I_{i} \sim 25 \mathrm{~km}$ considering Kerr nonlinear coefficient $\gamma=1.1(\mathrm{~W} \cdot \mathrm{km})^{-1}$; so $L_{N L}$ is comparable with the laser length $L=40 \mathrm{~km}$.

To conclude, in this paper we experimentally studied the interactions of spectral components within a multiwavelength radiation of the random fiber laser by simultaneously measuring the intensity dynamics for pairs of generation lines. The resulted Pearson correlation function contain a distinct correlation peak, which position is found to be linearly dependent on the spectral distance between studied lines. It allows us to locate a particular spatial location over the random fiber length where the interactions take place. Comparison with numerical simulations, based on the balance model, proves that such spatial localization is attributed to correlations appeared due to transfer of pump fluctuations to generation waves. We believe the presented method may be further developed as a powerful tool to study interactions in long fiber laser systems.

Funding. Russian Foundation for Basic Research (1632-60184, 17-02-00929).

Acknowledgment. Authors would like to acknowledge S.I. Kablukov for valuable discussions. I.D.V is supported by Russian Foundation for Basic Research (16-32-60184). O.A.G. and D.V.C. are supported by Russian Foundation for Basic Research (17-02-00929).

\section{References}

1. E. G. Turitsyna, S. V. Smirnov, S. Sugavanam, N. Tarasov, X. Shu, S. A. Babin, E. V. Podivilov, D. V. Churkin, G. Falkovich, and S. K. Turitsyn, Nat. Photonics 7, 783 (2013).

2. L. Carpi and C. Masoller, Phys. Rev. A 97, 023842 (2018).

3. S. Randoux, P. Walczak, M. Onorato, and P. Suret, Phys. Rev. Lett. 113, 113902 (2014).

4. D. R. Solli, G. Herink, B. Jalali, and C. Ropers, Nat. Photonics 6, 463468 (2012).

5. J. M. Dudley, F. Dias, M. Erkintalo, and G. Genty, Nat. Photonics 8, 755-764 (2014).

6. M. Conforti, A. Mussot, J. Fatome, A. Picozzi, S. Pitois, C. Finot, M. Haelterman, B. Kibler, C. Michel, and G. Millot, Phys. Rev. A 91, 023823 (2015).

7. M. Guasoni, J. Garnier, B. Rumpf, D. Sugny, J. Fatome, F. Amrani, G. Millot, and A. Picozzi, Phys. Rev. X 7, 011025 (2017).

8. A. Picozzi, J. Garnier, T. Hansson, P. Suret, S. Randoux, G. Millot, and D. N. Christodoulides, Phys. Rep. 542, 132 (2014).

9. O. A. Gorbunov, S. Sugavanam, and D. V. Churkin, Opt. Lett. 40, 1783 (2015).

10. S. Randoux and P. Suret, Opt. Lett. 37, 500 (2012).

11.I. D. Vatnik, O. A. Gorbunov, and D. V. Churkin, Laser Phys. 24, 025103 (2014).

12.P. Walczak, S. Randoux, and P. Suret, Opt. Lett. 40, 3101 (2015).

13. A. E. Bednyakova, O. A. Gorbunov, M. O. Politko, S. I. Kablukov, S. V. Smirnov, D. V. Churkin, M. P. Fedoruk, and S. A. Babin, Opt. Express 21, 8177(2013).

14.S. Sugavanam, M. Sorokina, and D. V. Churkin, Nat. Commun. 8, 15514 (2017).

15.S. K. Turitsyn, S. A. Babin, A. E. El-Taher, P. Harper, D. V. Churkin, S. I. Kablukov, J. D. Ania-Castañón, V. Karalekas, and E. V. Podivilov, Nat. Photonics 4, 231 (2010).

16.I. R. R. González, B. C. Lima, P. I. R. Pincheira, A. A. Brum, A. M. S. Macêdo, G. L. Vasconcelos, L. de S. Menezes, E. P. Raposo, A. S. L. Gomes, and R. Kashyap, Nat. Commun. 8, 15731 (2017).

17.L. L. Ogorodnikov and S. S. Vergeles, Opt. Lett. 43, 651 (2018).

18.S. A. Babin, D. V. Churkin, E. V. Podivilov, Opt. Commun. 226, 329 (2003).

19. O. A. Gorbunov, S. Sugavanam, I. D. Vatnik, and D. V. Churkin, Opt. Express 24, 19417 (2016).

20.S. Sugavanam, Z. Yan, V. Kamynin, A. S. Kurkov, L. Zhang, and D. V. Churkin, Opt. Express 22, 2839 (2014).

21. J. Lee Rodgers and W. A. Nicewander, Am. Stat. 42,59 (1988).

22.D. V. Churkin, A. E. El-Taher, I. D. Vatnik, J. D. Ania-Castañón, P. Harper, E. V. Podivilov, S. A. Babin, and S. K. Turitsyn, Opt. Express 20, 11178 (2012). 